\title{
The insensitive foot. Northwick Park experience
}

\author{
N P J GEARY \& L KLENERMAN \\ Northwick Park Hospital and Clinical Research Centre, Watford \\ Road, Harrow, Middlesex HAl 3 UJ
}

Accepted for publication 2 February 1986

Summary The problems of the pathogenesis and management of plantar ulceration in diabetic subjects and patients with leprosy are compared. Use of the total contact cast for treatment of an ulcer and methods for prevention of recurrence by appropriate insoles in footwear are described.

\section{Introduction}

Neuropathic foot ulceration may occur due to diverse pathological conditions; leprosy, diabetes mellitus spinal dysraphism, trauma to peripheral nerves, lesions of the cauda equina or spinal cord. Despite the variety of pathogeneses, the essential cause of the ulceration is the same. The tissues of the foot are subjected to a level of physical insult above the threshold at which tissue destruction occurs, without producing sufficient discomfort to the subject to trigger a response which will prevent further damage.

These conditions are for the most part irreversible and in the case of diabetes may be progressive. It is almost always impossible to do anything about the threshold level of insult at which tissue damage occurs, or the threshold level of stimulus at which avoidance of further exposure to the physical insult becomes mandatory. It is however quite possible to reduce the level of physical insult to the foot below the threshold level for tissue damage by education of the patient, special casting techniques and modified footwear. Applying this principle, we have had great success in healing neuropathic ulceration and preventing its recurrence. Furthermore it is open to us to prevent ulceration in the first place by identifying those at risk and applying sufficient prophylactic measures to keep the level of insult sustained by the foot below the tissue damage threshold.

At Northwick Park, the brief of our research grant is to identify and quantify risk factors in diabetic foot ulceration. As in leprosy, these patients may develop progressive neurological destruction even when under treatment. The threshold of tissue damage in diabetes may be lowered due to vascular obstruction, and tissue resistance to infection may be reduced by compromised cellmediated immunity. Apart from these features the ulceration in leprosy and diabetes has many of the same characteristics and the same principles apply. 


\section{Risk factors}

Risk factors in the formation of diabetic ulceration are traditionally classified after Oakley et al.," according to the system they affect. It is preferable to think functionally and consider risk factors on an aetiological basis.

What are the factors which place our patients at risk of ulceration? (a) Raised sensory threshold of avoidance reflex. (b) Reduced threshold of tissue resistance to damage. (c) Increased physical insult to the foot.

\section{RAISED SENSORY THRESHOLD OF AVOIDANCE 'RFF LEX'}

Sensory neuropathy reduces the subjects awareness of impending tissue damage. This does not only apply to mechanical modalities like pressure and shear stress, but also to thermal injury. We have seen painless ulceration resulting from chilblains and frostbite in Indians with diabetic neuropathy walking in sandals in the snows of Harrow.

\section{REDUCED THRESHOLD OF TISSUE RESISTANCE TO DAMAGE}

It is an oversimplification to talk of a single threshold of tissue damage but the concept is useful. The amount of trauma the tissues will withstand before ulceration will depend not only on the direction of the force and the area over which it is applied, but also, as Brand demonstrated, ${ }^{6}$ on the duration, frequency, and recent history of other trauma.

Major vessel obstruction is a common contributory feature reducing the tissue damage threshold in diabetes but, in leprosy it will only be as common as in the normal population. Where it does occur it may seriously affect the prognosis.

Autonomic neuropathy is thought to produce A-V shunting of blood in the foot ${ }^{13}$ possibly at the expense of cellular nutrition. The associated loss of sweating produces a dry cracked foot offering plentiful portals for the ingress of opportunistic infection. The mildly bactericidal effect of sweat is lost.

Small vessel disease; opinion is divided as to whether microangiopathy occurs in the foot in diabetics in the same way as it does in the eyes and kidneys. All the features attributed to it can be explained by autonomic vascular shunting. There is no suggestion of microangiopathy in leprosy.

\section{INCREASED PHYSICAL INSULT TO THE FOOT}

Thermal injury has already been mentioned and is only more likely because of the sensory loss.

Mechanical injury can be resolved into two directions; perpendicular load which is at right angles to the skin surface, and shear load which is parallel to the skin surface.

It is applied force per unit area that is the important factor in both cases, "the perpendicular and shear pressures'. In these units shear has much more damaging effect on the tissues, but the magnitude of shear is less.

Motor neuropathy produces a specific cavus deformity of the foot with initially interphalangeal flexion and later metatarso-phalangeal hyperextension and dislocation. The protective load distributing fibro-fatty pad under the metatarsal heads is dislocated distally so it can no longer help to dissipate load as low pressure over a wider area.

This deformity, the pedal equivalent of the intrinsic minus hand, results in the loss of the load sharing function of the toes.

In the normal individual, as the heel lifts in walking, the toes take over the load bearing function of the heel so the area of contact remains large. In the neuropathic deformity, when the heel lifts, the metatarsal heads only, divested of their protective fibro-fatty padding, transmit the body 
weight at much higher pressure. In severe cavus deformity there may be so much mid-foot flexion that the patient cannot get his heels to the ground at all.

The synovial sheaths around the flexor tendons to the toes normally provide an interface for the dissipation of shear stresses under the foot. The immobility of the toes means that the flexor tendons do not move, and all shear stresses are experienced by the thin skin now lying under the metatarsal heads.

The increased loading of the metatarsal heads causes the rays to spread producing a wide forefoot. If this wide forefoot is pushed into a normal shoe ulceration of the sides of 1st and 5th metatarsals may result. Pressure between the dorsally subluxed toes and the upper of the shoe may produce ulceration of the dorsum of the toes.

It should be noted that pressure applied to a convex surface tends to act cumulatively so that the pressure beneath the surface is greater than the applied pressure. This amplification is greater the tighter the curvature of the surface. ${ }^{5}$ Hence the sides of metatarsal heads and dorsa of the toes are particularly vulnerable.

Common orthopaedic foot deformities may afflict neuropathic patients like anyone. A bunion which may be subjected to pressure from the upper and hallux rigidus will result in tremendous pressure under the interphalangeal joint of the great toe.

Fixed flexion deformity at knee, hip or ankle may contribute to increased forefoot loading as the patient may not be able to get the heel on the ground except in very awkward postures. Bauman et al.' demonstrated the increased forefoot load in a case of 'dropped foot'.

\section{Risk evaluation}

How have we attempted to quantitatively assess the degree to which patients are affected by these risk factors?

\section{HISTORY}

An attempt has been made to estimate the amount of standing and walking each patient does and what loads are carried as magnitude duration and frequency of load are important.

\section{CLINICAL EXAMINATION}

Simple examination is valuable in assessing some of the problem features: overweight, hyperkeratosis in response to increased loading under the metatarsal heads, fissuring of the hyperkeratotic rim around the heel, pressure under the tips of clawed toes which if the nails are too long will be transmitted to the nail bed causing sub-ungual abscesses. Pressure on the sides of the metatarsal heads and the dorsa of the toes is best detected by examining the feet with the shoes. The distal medial corner of the great toe nail is a common site of bother. Dermatophyte infections may be an important portal of infection.

\section{RAISED THRESHOLD OF AVOIDANCE 'REFLEX' - SENSORY TESTING}

The biothesiometer is an electric vibrator with a controllable and calibrated amplitude of vibration. Excellent control data for age variation has been published. ${ }^{4}$ Most of our cases of neuropathic ulceration have a vibration threshold outside two standard deviations with none less than one standard deviation from the mean. A more accurate indication is expected from this method by the completion of this study with the inclusion of greater numbers and the calculation of 'variance' for each result. Unfortunately, age related control data is available for this machine only for the medial malleolus and great toe. 
The Semmes Weinstein Hairs ${ }^{10}$ are a modification of the Von Frey horsehairs using nylon fibres mounted in acrylic handles. If the fibre tip is pressed at right angles to the skin so that the fibre is maximally bent yet only touching the skin at the tip, the force applied to the skin will be constant, independent of minor movements of the hand and is repeatable. The force is related to the diameter of the fibre. It is possible to measure the sensory threshold by determining the finest fibre that can be perceived at a point. By repeating this determination at a matrix of points (in our case every square centimetre) it is possible to build up detailed sensory threshold maps of the foot. To do this with all 20 fibres in the set would be very tedious. Workers at the National Hansen's disease centre, in Carville, ${ }^{3}$ have shown that 1 fibre represents normal threshold of sensation and 1 represents the threshold of protective sensation. They advocated the use of a 3rd fibre, the thickest in the set that would still bend, to represent severe sensory loss. Thus four levels of sensory threshold can be determined; normal, loss of protective sensation, severe sensory loss (only feels the thickest fibre) and very severe sensory loss (cannot even feel the thickest fibre).

This mapping has been completed in 23 cases of ulceration and in 13 control cases with varying degrees of neuropathy. The results are still undergoing analysis at the time of writing. Our results with diabetics are consistent with the Carville findings in leprosy. With the exception of a grossly vascular cause, ulceration does not occur in areas having protective sensation.

\section{REDUCED TISSUE RESISTANCE TO DAMAGE}

Autonomic function in the foot can be assessed by skin galvanometry but our information is that this test is difficult to control and unreliable. Instead we are looking at autonomic function in another area by testing the autonomic cardiac reflexes.

Sepsis plays an important part in ulceration. Swabs from the throat, perianal region and toe webs of 44 patients with neuropathy have shown much lower than expected carriage rate of staphylococci and streptococci. We would suggest that lack of sweating of the neuropathic foot may protect against such carriage. We would expect then the moist conditions of a dermatophyte web infection to favour superinfection by such organisms and work is under way to look into this.

Inflammation secondary to mechanical injury or infection lowers the tissue resistance to further damage. Such inflammation is accompanied by hyperaemia detectable accurately with thermography.

An AGA 780 Infrared Thermographic System coupled to a Pericolar Image Analysis computer was used to record infrared thermograms of the skin surface. Measurements were carried out in standardized environmental conditions, in a temperature controlled room maintained at $27^{\circ} \pm 0 \cdot 5^{\circ}$. Ulceration in the neuropathic foot was frequently associated with increased skin temperature around the ulcer necrotic area while cold ulcers were generally found in feet with an atherosclerotic component of the disease.

\section{INCREASED PHYSICAL INSULT TO THE FOOT}

The pedobarograph is a computerized quantitative method working on an optical principle, which we are using to measure the interface pressure between the bare foot and the floor during walking, ${ }^{2}$ at intervals of $40 \mathrm{~ms}$. We hope to be able to arrive at a figure above which there is a significant risk of ulceration.

A method has been described for measuring shear during gait "but we have no experience of this. The Carville microsphere sock is probably the only way of objectively indicating excessive dorsal and lateral pressure. At present we rely on clinical examination.

\section{Treatment}

In the presence of adequate blood supply and with adequate control of serious infection, neuropathic ulcers will heal if the causative insult is removed or diminished. 
In the case of lateral and dorsal ulcers, this usually means providing footwear to accommodate the foot without applying excessive shear and perpendicular pressure. It may mean removing a foreign body from the shoe. In the case of high plantar pressure due to neuropathic deformity of the foot, the load on the metatarsal area for instance can be distributed as low pressure over the entire plantar aspect of the foot by the application of a plaster cast which is the exact shape of the sole of the foot, a total contact cast. ${ }^{7.8}$ We apply this as a below knee cast with padding over the pretibial border, the malleoli and the toes only. The toes are enclosed to prevent access of foreign bodies to the cast and to prevent the toes striking furniture etc. A rockered sole is fitted well back under the pretibial border to allow the cast to tilt forward with the minimum of effort, without the toes striking the ground and increasing the forefoot load.

We believe that the cast from the ankle up to the knee functions not to share in load bearing as has been suggested by some, but to minimize shear by ensuring that the sole of the foot is always approximately at right angles to ground reaction vector, and resisting fore/aft motion in the cast. This type of cast has been very effective in healing plantar ulcers, usually within 6 weeks.

\section{Prevention of recurrence}

Recurrence can be prevented by achieving the same conditions in the shoes as in the cast. Following Tovey's ${ }^{12}$ work we made an insole accurately shaped to the sole of the foot. The supporting cradle is made of rigid compressed high density plastazote. Sinks are cut in the cradle under areas of high risk and these holes are filled with the closed cell foam neoprene, which will not bottom out. The whole insole is then covered with neoprene for high load conditions, or an open cell foam, poron or PPT for lighter loading. This can only be accommodated in an extradepth shoe. Some are available commercially, but many severe cases require bespoke manufacture to accommodate deformity. The shoe is usually given a rockered sole with a thirty degree toe clearance and the fulcrum of the rocker set half way between the pretibial border and the lst metatarsal head. This is necessary to prevent toe/ground contact of the trailing foot on stepping forward. Except in those important cases with fixed flexion deformities, the shoe is given a zero pitch, as if there was no heel. This allows more toe clearance with much less height.

Patients are advised to moisten the feet daily with Boots E45 cream to prevent cracking, and to inspect the feet daily for areas of redness and increased warmth.

\section{Prophylaxis}

Besides the obvious advise to reduce risk of injury to the foot: weight reduction, walking and standing less, walking slower with shorter steps, keeping the nails in good trim and the skin moist, daily foot inspection, checking the shoes for nails and foreign bodies attention is paid to footwear. All patients should have shoes which will accommodate the foot without applying pressure. In the presence of deformity this may require prescription footwear; stock extradepth or bespoke depending on severity. Patients whom on testing are found to have significant neuropathy without high plantar pressures are given extradepth shoes with plain poron or neoprene $8-\mathrm{mm}$ insoles depending on loading. Those with high plantar pressure are given these shoes with the total contact insoles described for prevention of recurrence. We have not yet found it necessary to give rockered soles to a patient at risk who has not had ulceration. This is probably because we are still arriving somewhat late on the scene and all severely at risk patients will already have had at least one ulcer.

\section{The future}

It is hoped to institute at risk screening of all our diabetics in the clinics as follows. 
Those with no palpable ankle pulses will have a doppler examination of their ankle vessels. Those with poor flow will be given shoes and plain poron/neoprene insoles.

All will be screened with a biothesiometer. Those in the at risk group, at present $>2$ standard deviations from the mean will have a pedobarograph measurement. Those without high pressure will have shoes as above. Those with high pressure will have sensory mapping of the pressure areas. Those with protective sensation in the at risk area would have total contact insoles without rockers, those without protective sensation would have both, plus very regular supervision. The place of EMG and autonomic testing has yet to be determined. Dermatophyte infections will be treated vigorously.

\section{References}

1 Bauman JH, Girling JP, Brand PW. Plantar Pressures and Trophic Ulceration, an Evaluation of Footwear. J Bone Jt Surg (Br), 1963; 45-B: 652-73.

2 Betts RP, Franks CI, Duckworth T. Analysis of Pressure and Loads under the Foot. Clin Phys Physiol Meas. 1980; 1(2): 101-24.

${ }^{3}$ Birke JA, Chief Physical Therapy Dept, National Hansens' Disease Center, Carville, LA. Personal Communication, May 1985.

${ }^{4}$ Bloom S, Till S, Sönksen P, Smith S. Use of a biothesiometer to measure vibration thresholds and their variation in 519 non-diabetic subjects. Br Med J, 1984; 288: 1793-5.

5 Brand PW. The diabetic foot. In: Diabetes Mellitus, Theory and Practice, 3rd edn. Ellenberg M, Rifkin H. (eds), New Hyde Park: Medical Examination Publishing Co. Inc., 1983; 829-49.

${ }^{6}$ Brand PW. Pathomechanics of Pressure Ulceration. In: Symposium on the Neurological Aspects of Plastic Surgery. Fredricks, Brock. (eds), St Louis: C V Mosby Co., 1978; 185-9.

7 Coleman WC, Brand PW, Birke JA. The Total Contact Cast. Journal of the American Podiatry Association, 1984; 74(11): 548-52.

${ }^{8}$ Helm PA, Walker SC, Pullium G. Total Contact Casting in Diabetic Patients with Neuropathic Foot Ulcerations. Arch Phys Med Rehab, 1984; 65: 691-3.

9 Oakely W, Caterall RCF, Martin MM. Aetiology and management of lesions of the feet in diabetes. Br Med J, 1956; 2: 953-7.

10 Semmes J, Weinstein S, Ghent L, Hans-Lukas T. Somatosensory changes after Penetrating Brain Injury in Man. Cambridge: Harvard University Press, 1960.

11 Tappin JW, Pollard J, Beckett EA. Method of measuring 'shearing' forces on the sole of the foot. Clin Phy's Physiol Meas, 1980; 1(1): 83-5.

12 Tovey FI. The manufacture of diabetic footwear. Diabetic Medicine, 1984; 1(1): 69-71.

13 Ward JD. The Diabetic Leg. Diabetologia, 1982; 22: 141-7. 